\title{
The Problem of Trope Individuation: A Reply to Lowe
}

\author{
[penultimate draft, to be published in Erkenntnis] \\ Markku Keinänen \\ Department of Behavioural Sciences and Philosophy \\ 20014 University of Turku \\ Finland \\ markku.keinanen@utu.fi \\ Jani Hakkarainen \\ School of Social Sciences and Humanities \\ 33014 University of Tampere \\ Finland \\ jani.hakkarainen@uta.fi
}

\begin{abstract}
This paper is the first trope-theoretical reply to E.J. Lowe's serious dilemma against trope nominalism in print. The first horn of this dilemma is that if tropes are identity dependent on substances, a vicious circularity threatens trope theories because they must admit that substances are identity dependent on their constituent tropes. According to the second horn, if the trope theorist claims that tropes are identity independent, she faces two insurmountable difficulties. (1) It is hard to understand the ontological dependence of tropes on substances. (2) The identity-conditions of tropes cannot be determinate, which threatens the determination of the identityconditions of substances. Our reply to the first horn of Lowe's dilemma is to deny the identity dependence of tropes. Yet we can avoid the second horn because our theory
\end{abstract}


can explain the ontological dependence of tropes on substances and the fullydetermined identity-conditions of both tropes and substances.

Keywords: trope, identity conditions, existential dependence 


\section{Introduction}

The problem of concrete particulars belongs to the basic problems of contemporary metaphysics, cf. Loux (1998, ch.3). It concerns the constitution of individual substances, i.e., concrete particulars that do not need other entities in order to exist. Amongst the nominalist answers to this problem - which do not assume universals -, there are two types of ontologies/category systems that postulate tropes (particular properties). On the one hand, individual substances may be construed as consisting of tropes and a substratum in which tropes inhere. On the other hand, substances can be mere bundles of tropes. Accordingly, the latter accounts are usually called the tropebundle theories of substances/concrete particulars.

E.J. Lowe presents a dilemma against the trope-bundle theories that takes the coherence of the pure trope construction of substances as its exact target. As will be seen just below, there is no easy way out of this dilemma for the trope-bundle view. Since we have previously defended a trope-bundle theory of substances, Lowe's dilemma is a serious objection to our account that needs to be answered. Neither has anybody, as far as we know, defended trope-bundle theory from the dilemma in print (not to speak of our type of trope-bundle theory) - even if Lowe has advanced his counter-argument twice in his publications (Lowe 1998, 205-9; 2003, 82-5). So this paper is the first trope-bundle-theoretical reply to Lowe.

According to Lowe $(1998,205-9)$, any attempt at determining the identityconditions of tropes leads to the following dilemma. The trope theorist must either admit that tropes are identity dependent on the individual substances ${ }^{1}$ (trope bundles) possessing the tropes, i.e., that the identity-conditions of tropes are given by means of the identity-conditions of individual substances or assume that tropes are not identity dependent on individual substances. Both of these alternatives lead to an impasse. If we adopt the first alternative, an unacceptable circularity of identity dependence ensues: tropes depend for their identity on the individual substance they constitute and the individual substance is identity dependent on its constituent tropes. On the other hand, if we choose the second alternative and assume that tropes do not depend for their identity on the individual substance, we encounter two serious problems. First, it is difficult to make sense of their ontological dependence on individual substances, which should act as their bearers. This problem concerns thus inherence: the relation between tropes and substances (a substance having a property). Secondly, 
we do not have any clear means of determining the identity-conditions of tropes (and their identity and diversity). Since individual substances as trope bundles depend for their identity on their constituent tropes, this threatens to make individual substances entities which have no determinate identity-conditions at all - a result that should be avoided.

Lowe considers this dilemma as the decisive argument against trope theories: according to him, the only way out of it is to reject the trope-bundle theory of individual substances. According to Lowe, individual substances are categorially simple entities that have determinate identity-conditions. Tropes (or, modes) are attribute-like individual accidents of substances, not their parts. Each trope is identity dependent on the individual substance in which it inheres if it has determinate identity but the converse does not hold. ${ }^{2}$

In this article, we defend a specific trope-bundle theory, the Strong Nuclear Theory (SNT) against Lowe's criticism. We argue that the trope theorist can consider tropes as entities not identity dependent on any other entity but avoid the problems taken up by Lowe. First, the advocate of the SNT can make perfect sense of tropes as existentially dependent on individual substances without assuming that tropes depend for their identity on them. Secondly, we argue that the trope theorist is entitled to maintain that each trope has determinate identity-conditions without making it identity dependent on any further entity. The identity and individuation of tropes is primitive: there is no metaphysical explanation or ground for them. ${ }^{3}$

Before we begin our argument, a methodological remark is required. Primarily, we discuss the metaphysical question of individuation in this article: what individuates tropes in the world? How individuation should be understood is a metaphysical problem in its own right. In this paper, we take individuals to be entities that are countable and that have determinate identity. So we understand individuation to involve the following two questions. (1) What makes single tropes unities (genuinely one entity) so that there is a fact to the matter, how many tropes there are in some location? (2) Why are tropes not identical with each other and entities of other kinds: why is there a distinction between them and other tropes or entities of other kinds - synchronically or diachronically? ${ }^{4}$ Or conversely, why are tropes $x$ and $y$ identical? Lowe puts the latter point more specifically in terms of "identityconditions" and "criteria of identity", both at a time and over time. In the case of identity between tropes, the identity-conditions of tropes mean the truth-conditions of 
identity statements of the form 'trope $x$ is the same trope as trope $y^{\prime}$. When these statements are informative (non-trivial and non-circular), they provide criteria of identity for tropes. (Lowe 1998, 59) ${ }^{5}$

As does Lowe, we think the metaphysical problem of individuation should be clearly distinguished from the more epistemic or cognitive problem of identification: how we are able to identify tropes (actively) as countable and non-identical with each other or entities of other kinds (Lowe 2003, 75). ${ }^{6}$

This article has five parts. In section 2, we first concentrate on the first horn of the dilemma, where it is assumed that tropes depend for their identity on substances. After the rejection of this assumption, we take up the second horn that tropes are not identity dependent in section 3 . We argue that the trope theorist can accept their identity independence and avoid the criticism Lowe presents. In section 4, we briefly explain our account of trope identification and discuss problems regarding it such as trope piling. Finally, section 5 concludes the article by pulling the strings together and summarising.

\section{Tropes as Identity Dependent Existents}

Lowe seems to consider the argument against trope theories based on the first horn fairly decisive for two reasons. First, according to him, the prima facie best trope theories entail that every property trope is existentially dependent on individual substance $i$ in which it inheres, i.e., that $t$ is rigidly dependent on $i$ (Lowe 1998, 206). Secondly, Lowe $(1998,146)$ argues that the proper clarification of ontological dependence of particular entity $t$ on some definite substance $i$ is in terms of identity dependence. With certain qualifications, identity dependence entails rigid dependence. ${ }^{7}$ Importantly, the converse does not hold because some entities can be rigidly dependent on certain other entities without depending for their identity on them. Most notably, identity dependence is anti-symmetric while rigid dependence is not (Lowe 1998, 146, 150). Lowe's example is that Socrates' life and Socrates seem to be mutually existentially dependent although we appear to have strong intuition that Socrates' life rather than Socrates is the truly dependent existence here (Ibid. $145)$ 
If entity $e$ is identity dependent on entity $f$, the identity-conditions of $e$ are given in terms of the identity-conditions of $f$. According to the traditional broadly Aristotelian conception, property tropes (or, modes) are identity dependent in this sense: two exactly similar tropes are distinct if and only if they are properties of distinct individual substances. That, e.g., two exactly similar shape tropes are properties of distinct substances $a$ and $b$ seems to make them two distinct shape tropes. Accordingly, Lowe $(1998,147)$ characterizes identity dependence as follows:

[ID]: Entity $e$ is identity dependent on entity $f$ is to say that which entity of its kind $f$ is metaphysically determines which entity of its kind $e$ is.

Thus, for instance, the identity of individual substance $i$ fixes (or, metaphysically determines) the identity of the shape trope $t$ of $i$ and the identity of a set is fixed by the identity of its members. As was seen just above, the relation of identity dependence is anti-symmetric for Lowe: if entity $e$ is identity dependent on distinct entity $f, f$ cannot be identity dependent on $e$. He explains the anti-symmetry as follows. If the identity of $f$ metaphysically determines the identity of distinct entity $e$, the identity criteria of $e$ are given by means of the identity criteria of $f$. Since each entity must have non-circular identity criteria, the identity criteria of $f$ cannot be given by means of the identity criteria of $e$. Thus, $f$ cannot be identity dependent on $e$. According to Lowe, substances are not identity dependent on any other entity and primitive substances form examples of entities which do not have any informative diachronic criteria of identity (Lowe 1998, 150, 169-70).

Now, we can formulate Lowe's $(1998,206)$ first argument against trope theories as follows:

[P1]: According to the trope-bundle theories, individual substances are bundles of property tropes. Tropes are parts of a substance, the entities constituting the substance.

[P2]: According to the best trope theories, tropes are identity dependent on their bearers, i.e., individual substances. 
[P3]: If individual substances are trope bundles, they are identity dependent on some of their trope constituents.

[P4]: Identity dependence is anti-symmetric: if $e$ and $f$ are distinct entities and $e$ is identity dependent on $f, f$ cannot be identity dependent on $e$.

Thus, an individual substance both depends for its identity on some of its constituent tropes and all constituent tropes of the substance are identity dependent on it (by [P2] and [P3]). However, this contradicts the anti-symmetry of identity dependence ([P4]). Consequently, we must reject either some of the premises [P1][P4] or the trope-bundle theory of substances (SNT).

It seems difficult to reject any of the premises [P1]-[P4]. [P1] correctly characterizes all trope-bundle theories of substance. Plausibly, as a trope bundle, an individual substance is identified by means of its necessary trope-constituents and the identity-conditions of the substance are given by means of the necessary tropes. ${ }^{8}$ Hence, the substance is also identity dependent on its necessary trope constituents ([P3]). The truth of [P4] is a direct consequence of the above characterizations of identity dependence in combination with the reasonable requirement that entities cannot have circular identity-conditions. Finally, the traditional conception of tropes as accidents (or modes) of individual substances entails that tropes depend for their identity on individual substances. [P2] results from the attempt to accommodate this conception by the trope-bundle theory.

Since the acceptance of [P1]-[P4] leads to trouble, the trope theorist cannot accept all of these premises. We consider [P1] as undeniable. Moreover, [P3] and [P4] are plausible if we accept the notion of identity dependence and recognize the close link between identity dependence and the determination of the identityconditions of entities. The trope theorist does not have any clear prima facie reason to deny the acceptability of these notions and the close link between them. Hence, the only viable option left for the trope theorist is to deny [P2]: tropes are identity dependent on substances. That is the topic of the next section.

\section{Tropes as Identity Independent Existents}


In this section, we defend the following thesis: property tropes are identityindependent existents, i.e., they are not identity dependent on any distinct entity. Given that trope $t$ exists, it has identity on its own independent of the identity of any distinct entity (e.g., a substance or a space-time point). We deal with the two main complaints that Lowe $(1998,207)$ presents about this thesis: first, that if a trope is not identity dependent on an individual substance, it is difficult to understand its status as

a dependent existent and as a property of some substance. Secondly, we do not have any clear means of determining the identity-conditions of tropes (and their identity and diversity). Since as trope bundles substances are identity dependent on their constituent tropes, this threatens to make individual substances entities having no determinate identity-conditions. As we defend a specific trope theory, the SNT, for these complaints, we only show that our theory can deal with them.

\subsection{The Identity-Conditions of Tropes}

Let us begin with the second complaint. According to the SNT, every trope has fully-determined identity. This identity is primitive or ungrounded in every respect. As well as the particularity of tropes (see note 5), it is a brute fact that tropes are unities (as simples) that are distinct from each other and entities of other kinds, both synchronically and diachronically. Since tropes are countable as unities, it is fully determinate how many tropes there are in some particular location. ${ }^{9}$ Recall that by the identity-conditions of items of a kind $K$, Lowe means the truth-conditions of identity statements of the form " $x$ is the same $K$ as $y$ ". These kinds of statements about tropes have fixed truth-conditions. For example, the statement "- $e$ trope $t$ is the same as - $e$ trope $u$ " has determinate truth-conditions because there is a fact to the matter whether they are identical.

Hence, tropes have fully-determined identity-conditions - contra Lowe. This does not entail, however, that tropes have informative criteria of identity (although the statements about identity of tropes have fixed truth-value); generally, the abovementioned statements of tropes are not informative (non-trivial) because the identityconditions of tropes are not given by the identity-conditions of any distinct entity. In this respect, too, tropes resemble Lowe's primitive substances, which do not have any criteria of diachronic identity (Lowe 1998, 169-70). 
The core point is that the identity of tropes is not grounded in the identity of other tropes or entities of other kinds. Rather, the identities of entities of other kinds are grounded in the primitive identity of tropes. In a word, tropes are the basic building blocks of the universe in the SNT: entities of other kinds are composed of them and their primitive identity determines the identity of these other entities. Still it does not follow from the primitive identity of tropes that simple substances (substances that do not have other substances as their proper parts) do not have determinate identity-conditions. Their identity-conditions are provided by tropes independently of substances and these conditions are determinate.

In the SNT, every simple substance has one or many tropes that are necessary to it. They are called "nuclear tropes", which are distinguished from contingent tropes. Nuclear tropes provide the identity-conditions for a simple substance both synchronically, diachronically and counterfactually: for instance, the simple substance persists as long as there are these nuclear tropes necessary to it (Keinänen \& Hakkarainen 2010, 126).

Lowe does not and will not accept our solution. He rules it out that the individuation of tropes is primitive: tropes are not identity independent existents if they have, as many tropes do, determinate identity (and identity-conditions). As far as we can see, the only reason Lowe offers against this primitiveness is an eliminative argument, which is supposed to show that his position is the only option: if modes (tropes) have determinate identity, they are identity dependent. Lowe asks: "if tropes are not identity dependent upon their possessors, what does determine their identity or diversity?" (Lowe 1998, 207) Since two exactly similar tropes can occupy the same spatio-temporal location, Lowe rejects the alternative that the location could determine their identity (Lowe 1998, 207-208). He finds no further alternative way in which tropes could have determinate identity, e.g., that the trope identity is primitive.

Our reply to this counter-argument is that Lowe does not actually argue against the primitivity of the identity of all tropes; he does not really consider that position. He just does not find it as a viable option because his view is that there are identitydependent modes. If this position of Lowe is somehow motivated by intuitions concerning trope identification, our distinction between epistemic identification and metaphysical individuation is helpful. We will show in section 4 that we can identify tropes by means of other entities without rendering them identity-dependent. ${ }^{10}$ 


\subsection{Tropes as Properties of Objects}

According to Lowe's first complaint in the second horn of his dilemma, if a trope is not identity dependent on a substance, it is difficult to understand its status as a dependent existent, as a property of some individual substance. According to Lowe $(1998,207)$, if tropes are not identity dependent on individual substances, the trope theorist "needs to explain in what sense tropes are really ontologically dependent entities". As Lowe points out, it is desirable that the trope theorist can rule out both single free-floating tropes (tropes without substances/as substances) and trope migration: trope $t$ first is a property of substance $i$ and later ceases to be a property of $i$ and becomes instead a property of a different substance.

In order to answer the first complaint in the second horn, it is necessary to present some basic features of the SNT and how it differs from other trope theories. The SNT starts with the assumption that simple substances are powerful particulars: all of their intrinsic features are basic physical quantities (determinate masses, charges, etc.) and dispositional features of objects, i.e., necessarily, they furnish an object with certain definite causal powers. ${ }^{11}$ Moreover, simple substances divide into natural kinds and many of such determinate features are necessary to a certain kind of substance (e.g., electron). According to the SNT, tropes determine the causal powers of a simple substance, which are basic physical quantities (such as charge $-e$ of the electron). ${ }^{12}$

Thus, the SNT adopts a "sparse theory of tropes" (cf. Campbell 1990, 24-25): tropes determine the features of simple objects. According to the SNT, simple substances that are bundles of tropes, complex substances are composed of simple substances (Keinänen 2011, 447). We do not take any further stance of the composition of complex substances, which have other substances as their proper parts. Given that there are complex substances, their features are, in many cases, determined by the features of their proper parts, and eventually, by the features of simple substances. ${ }^{13} \mathrm{We}$ do not commit ourselves to quality tropes such as particular colours or shapes: for instance, there is no need for monadic shape tropes as the shapes of macroscopic objects are determined by their having certain kinds of proper parts in a certain arrangement. ${ }^{14}$

The SNT and Campbell's (1990) trope theory share the feature that tropes can be spatio-temporally compresent, but one of their differences is that the SNT extends 
this to simple substances: two simple substances can be in the same place at the same time (Keinänen (2011, 435ff.), see also note 27). A good prima facie example of a simple substance is a down quark or an electron. The nuclear tropes such as a rest mass trope of $9.10938215 \times 10^{-31} \mathrm{~kg}$ and a charge trope of $-e$ determine the necessary features of the electron, which are determinate physical quantities.

On this basis, we can construct an analysis of inherence while maintaining that tropes are identity independent. According to the SNT, tropes are ontologically dependent: they are all rigidly dependent on certain further tropes. ${ }^{15}$ The SNT does not introduce identity dependence, or alternatively, inherence as a primitive but takes strong rigid dependence as a primitive formal relation by means of which we can analyse inherence. ${ }^{16}$ Take any trope $t$. It is strongly rigidly dependent (SRD) on the nuclear tropes of a simple substance. Consequently, necessarily, if trope $t$ exists, every nuclear trope $u$ of the substance exists. The nuclear tropes of a simple substance are strongly rigidly dependent on each other.

According to the SNT, every simple substance has one or more nuclear tropes necessary to it. They determine kind K (e.g., electron) to which the simple substance belongs. As tropes of a substance of kind $\mathrm{K}$ all of the tropes of a simple substance are strongly generically dependent (SGD) ${ }^{17}$ on tropes falling under certain distinct (highest) determinables. There must be tropes falling under each of these determinables rigidly dependent on the nuclear tropes. Many of such tropes are nuclear tropes, but some of them might be one-sidedly rigidly dependent on the nuclear tropes. These tropes are merely contingent to a simple substance.

There are three substantial differences between the SNT and Peter Simons' (1994) Nuclear Theory, from which the SNT has been developed. The first is that the SNT makes all tropes (also such nuclear tropes that suffice to constitute a simple substance) generically dependent on tropes falling under certain distinct determinables. Thus, every substance must contain tropes falling under distinct determinables: for instance, there cannot be simple substances formed by one or more mass tropes. Secondly, according to the SNT, every nuclear trope of a simple substance must fall under a distinct (highest) determinable. So there is only one charge and mass trope composing the electron, for example. Thirdly, unlike Nuclear Theory, the SNT constrains the location of the tropes constituting a substance (cf. below). 
As every trope of a simple substance is SRD on every nuclear trope of that substance and the nuclear tropes of substances are necessary to them, all its tropes are bound to a simple substance by the formal relation of strong rigid dependence. Therefore, the following principle holds: trope $t$ is a part of simple substance $i$ iff $t$ is strongly rigidly dependent only on the nuclear tropes of $i$ (Keinänen 2011, 438). By contrast, simple substances are strongly independent particulars, i.e., particulars that are not strongly rigidly dependent on any distinct entity. Since simple substances are trope aggregates in which all the rigid dependencies of the constituent tropes are fulfilled, they are not strongly rigidly dependent on any distinct entity. ${ }^{18}$

Strong rigid dependence excludes dependence on parts, which the tropes of a simple substance are for it. Unlike Lowe, the SNT does not rule it out that simple substances are dependent existents; they are weakly rigidly dependent on their nuclear tropes because weak rigid dependence admits of dependence upon parts. Moreover, we can reduce the identity dependence of simple substances on their trope parts to proper parthood and mutual rigid dependence: substance $i$ is identity dependent on trope $t$ iff $t$ is a proper part of $i$, rigidly dependent on $i$ and $i$ is rigidly dependent on $t .{ }^{19}$ Lowe is sceptical of substances having such necessary parts and he does not discuss this vital distinction between strong and weak rigid dependence upon which the SNT builds. ${ }^{20}$

One of the implications of what was said just above is that every trope must be a constituent of some simple substance composed of two tropes falling under distinct determinables (tropes of distinct physical quantities) at least. ${ }^{21}$ So, substances consisting of one trope and free-floating tropes without substance are ruled out in the SNT. It also excludes trope migration between distinct substances: every property trope is rigidly dependent on the nuclear tropes of exactly one substance. This is Lowe's understanding of trope migration. ${ }^{22}$

As was remarked just above, the SNT differs from Nuclear Theory in the following third respect: it is a consequence of the SNT that each simple substance is necessarily co-located with its nuclear tropes. We have also argued elsewhere that the contingent tropes of a simple substance are necessarily co-located with the substance at every moment of their existence (Keinänen \& Hakkarainen 2010, sec.2).

Consequently, the SNT analyses inherence as follows: trope $t$ is a property of $i$ iff $t$ is rigidly dependent on $i$, a proper part of $i$, and its spatio-temporal location is a (proper or improper) part of the spatio-temporal location of $i$. Thus, tropes cannot 
migrate by changing their location relative to the simple substance; a trope must be where and when the simple substance possessing it is.

The SNT is a qualitatively economical ontological theory, which also reduces the number of primitive formal relations in comparison with Lowe's Neo-Aristotelian position. In addition to the analysis of inherence, we can propose an analysis of identity dependence by means of proper parthood and rigid dependence: tropes are identity independent existents. There are no identity dependencies between mereologically simple entities. All identity dependencies obtain between entities and their necessary proper parts. In the SNT, existential dependencies are rigid or generic dependencies and we need not rely on the problematic notion of essence. However, these formal relations are restricted to the sphere of contingent existents. ${ }^{23}$

Finally, one of the consequences of this view is that we disagree with those metaphysicians who individuate tropes by means of their spatiotemporal location (cf. Campbell 1981; Schaffer 2001). ${ }^{24}$ J. Schaffer (2001, 248-249), for instance, advocates the following spatiotemporal individuation principle (SI): $\mathrm{x}$ and $\mathrm{y}$ are distinct tropes if they are either not exactly resembling, or at distant locations (Distance $(\mathrm{x}, \mathrm{y})>0$ ). We have two main reasons for disagreeing on the (SI). First, as was seen above, the SNT allows of co-located simple substances and also co-located exactly similar tropes, while the (SI) rules them out. Second, and independent of the SNT, it is hard to combine the (SI) with the claim that tropes are identity independent existents. Even if tropes are not individuated by space-time points (which would compromise their identity independence), it is difficult to individuate tropes by means of their spatio-temporal relations to other tropes and maintain their identity independence.

Schaffer also claims that the primitive identity is "epistemologically problematic" as we cannot perceive primitively distinct tropes (2001, 249). However, it depends on our exact account of trope identification how problematic the separation of the distinct tropes actually is. ${ }^{25}$ This is the subject of the next section.

\section{Identification of Tropes}

According to our proposal, we can identify tropes (and have access to their identity-conditions) because tropes are, necessarily, properties of simple substances 
belonging to some kind $\mathrm{K}$. According to the SNT, every trope $u$ is a property of some substance of kind $\mathrm{K}$. As was seen just above, it also states that $u$ is necessarily colocated with certain kinds of tropes (the tropes of certain determinate kinds) determining kind $\mathrm{K}$ to which that substance belongs. Moreover, we can rule out several tropes that fall under a single determinable and are properties of a substance at a certain moment of time T. Consequently, the following principle [IA] is valid:

[IA]: If trope $u$ is a property of a substance of kind $\mathrm{K}$ and there is exactly one substance of kind $\mathrm{K}$ in the same spatio-temporal location as trope $u$, then trope $u$ is a property of that substance.

In order to see how this principle can be used in identifying tropes, let us first discuss a difficult case where it cannot be employed. It is difficult to identify the colocated substances belonging to the same kind $\mathrm{K}$ (e.g., the distinct co-located electrons). As C. Daly has pointed out, the trope constituents of such substances are equally difficult from the perspective of trope identification (Daly 1997, 155-6). [IA] cannot be applied to them. Hence, if there is object piling, i.e. several substances of the same kind $\mathrm{K}$ in the same location, there is also trope piling (exactly similar tropes in the same location) in the SNT. For instance, if there are two electrons in the same location, there are also two co-located $-e$ tropes. In that case, identifying these two tropes is not possible by [IA]. ${ }^{26}$ We do not discuss cases of this type any further. ${ }^{27}$

With regard to other scenarios that do not involve object piling, we can use [IA] for identifying tropes. The tropes occupying the distinct spatio-temporal locations are distinct. Given that [IA] holds, we can identify the different tropes in some spatiotemporal location: first, we distinguish the different kinds of simple substances in a spatio-temporal location (this is, of course, a matter of empirical investigation). Secondly, by applying [IA], we distinguish the different tropes in that location: tropes are properties of a certain kind of simple substance in some particular location.

The manner of identifying tropes that we suggest does not entail any circularity in the individuation of tropes. Our approach tells how we are able to identify tropes in a particular location. It does not provide any informative identity-conditions of tropes but rather relies on tropes already having primitive identity. The moot point is that every trope is rigidly dependent on certain kind of substance $i$ and necessarily colocated with that substance at each moment of its existence but not identity dependent 
on it. The tropes having certain identity-conditions are accessible to us through how they function as constituents of the world: as constituents of certain kinds of trope bundles (simple substances). However, the ways we identify tropes do not have any consequences with regard to their identity-conditions.

Simple substances are identity dependent on their nuclear tropes and thus identified by means of them. However, our approach does not entail any circularity in trope identification. In order to identify trope $u$ all that we need to know is that $u$ is the property of a substance of kind $\mathrm{K}$. Consequently, $u$ is necessarily co-located with nuclear tropes belonging to certain determinate kinds (e.g., a charge trope $-e$ and a mass trope of $\mathrm{M}$, etc.), which are tropes necessary to a simple substance at every moment of its existence. However, in order to identify trope $u$, we need not recourse to any particular trope or particular substance. As identification is done by certain kinds of (simple) substances and tropes, it is generic.

An advocate of the SNT agrees with Lowe that tropes are, necessarily, properties of simple substances - they cannot float free or migrate between distinct substances. Still tropes are parts of simple substances. The identity-conditions of simple substances are determined by their nuclear tropes. Nuclear tropes are accessible to us because they are necessarily constituents of a certain kind of trope nucleus. The contingent tropes are accessible to us because they are necessarily constituents of a certain kind of substance.

\section{Conclusion}

This paper is a novel, dependence-trope-bundle-theoretical reply to E.J. Lowe's argument against the trope-bundle theories of individual substances in the case of simple substances. Lowe presents the dilemma that the advocate of a trope-bundle theory must admit either that tropes are identity dependent on the individual substances that possess them or that they are identity independent entities. The first horn of the dilemma leads to a vicious circularity: tropes depend for their identity on the substances possessing them and these substances are equally identity dependent on the tropes because individual substances are bundles of tropes in the trope-bundle theories. On the other hand, if tropes are taken not to depend for their identity on substances or any other distinct entity, the trope-bundle theory faces two 
insurmountable difficulties. (1) If tropes are not identity dependent on their substances, it is hard to understand their ontological dependence on these substances; the ontological dependence in this case appears to be exactly identity dependence. (2) The identity-conditions of tropes cannot be determinate, which threatens the determination of the identity-conditions of substances.

Our way out of this dilemma is twofold. First we deny the first horn that tropes depend for their identity on simple substances. Secondly, we explain how this is possible without falling into the trap that we cannot account for the ontological dependence of tropes on simple substances and that simple substances do not have fully-determined identity-conditions.

The fundamental points of our explanation are the following: metaphysical individuation should be distinguished from epistemic identification and the individuation of tropes is primitive or metaphysically ungrounded. In our theory, tropes are basic physical quantities. It is a brute fact that they are true unities (countable) differing from other tropes (quantities) and entities of other kinds (having identity-conditions) like simple substances, which are bundles of tropes. This does not lead to the indeterminacy of the identity-conditions of simple substances. On the contrary, nuclear tropes necessary to a simple substance provide fully-determined identity-conditions for it.

On this basis, our account can also make sense of the ontological dependence of tropes on simple substances. Every substance has one or more nuclear tropes, which are mutually rigidly dependent entities. Every trope is strongly rigidly dependent on the nuclear tropes of certain simple substance $i$, and thereby strongly rigidly dependent on $i$ and a proper part of $i$. Simple substances are dependence closures of tropes. Tropes depend for their existence on the substance possessing them but have primitive identity, i.e., are identity independent existents. Rather, since a simple substance is weakly rigidly dependent on its nuclear tropes (i.e., the tropes necessary to it), the nuclear tropes determine its identity. Still, the rigid dependence of tropes on the nuclear tropes of a certain substance suffices to guarantee that they can be identified as properties of a certain kind substance. This gives us resources to deal with such scenarios of trope piling, discussed in the literature, where there is one substance or several substances of distinct kinds involved. The basic idea is that tropes can be identified by the kinds of substances whose constituents they are. 


\section{Acknowledgements}

For comments of the earlier drafts, we would like to thank the four referees of Erkenntnis as well as Fabrice Correia, Antti Keskinen, Philipp Keller, E.J. Lowe, Arto Repo, and the audiences at the University of Geneva, University of Tampere and University of Turku. This work has been financially supported by the Academy of Finland research projects "Tropes, Change and Dispositional Essentialism" and "British Empiricism and Trope Nominalism".

\section{References}

Campbell, K.K. (1981). Metaphysic of Abstract Particulars. Midwest Studies in Philosophy 6, 477-488.

Campbell, K. K. (1990). Abstract Particulars. (Oxford: Basil Blackwell)

Daly, C. (1997): Tropes. (In D. H. Mellor \& Alex Oliver (Eds.). Properties (pp. 140-159).

Oxford: Oxford University Press)

Ehring, D. (2011). Tropes: Properties, Objects, and Mental Causation. (Oxford: Oxford University Press).

Ellis, B. D. (2001). Scientific Essentialism. (Cambridge: Cambridge University Press).

Fine, K. (1995). Ontological Dependence. Proceedings of the Aristotelian Society 95, 269290.

Keinänen, M. (2011). Tropes - the Basic Constituents of Powerful Particulars? Dialectica $65,3,419-450$.

Keinänen, M. \& Hakkarainen, J. (2010). Persistence of Simple Substances. Metaphysica $11,2,119-135$.

Loux, M. (1998). Metaphysics. A Contemporary Introduction. (London \& New York: Routledge).

Lowe, E. J. (1998). The Possibility of Metaphysics - Substance, Identity and Time. (Oxford: Clarendon Press).

Lowe, E. J. (2003). Individuation. (In M. Loux \& D. Zimmerman (Eds.) The Oxford Handbook of Metaphysics (pp. 75-95). Oxford: Oxford University Press).

Lowe, E.J. (2006). The Four-Category Ontology. (Oxford: Clarendon Press). 
Lowe, E.J. (2009). Ontological Dependence. In Edward N. Zalta (Ed.), The Stanford Encyclopedia of Philosophy, Fall 2009 edition, Retrieved from September 12, 2009 from http://plato.stanford.edu/entries/dependence-ontological/

Maurin, A-S. (2002). If Tropes. (Dordrectht: Kluwer Academic Publishers).

Morganti, M. (2009). Tropes and Physics. Grazer Philosophische Studien 78, 185-205.

Mumford: S. (2006). The Ungrounded Argument. Synthese 149, 71-89.

Schaffer, J. (2001). The Individuation of Tropes. Australasian Journal of Philosophy 79, 2, 247-257.

Simons, P. M. (1987). Parts - a Study in Ontology (Oxford: Clarendon Press).

Simons, P. M. (1994). Particulars in Particular Clothing - Three Trope Theories of Substance. Philosophy and Phenomenological Research LIV, 3, 553-75.

Simons, P. M. (1998). Farewell to Substance - a Differentiated Leave-Taking. Ratio XI, 235-251.

${ }^{1}$ Lowe $(1998,58)$ uses the term "individual object" for all entities that have determinate identityconditions and form countable unities (are countable). Substances are individual objects that are not ontologically dependent on further entities (Lowe 1998, ch.6). They also have the traditional function to act as the bearers of property tropes (modes).

${ }^{2}$ Lowe allows of tropes or modes that do not have determinate identity. As such, they are not identity dependent on their bearers. This does not mean, however, that tropes or modes are ontologically independent because they are adjectival of their bearers (ways those bearers are) (Lowe 1998, 79-83).

${ }^{3}$ According to K. Campbell and D. Ehring, too, the individuation of tropes is "basic and unanalysable", or "primitive" as Ehring puts it (Campbell, 1990, 69; Ehring, 2011, 76-91). However, our position differs from them essentially because in their theories tropes are not strongly rigidly dependent (cf. note 6 for definition) on any entity (Campbell 1990, 21; Ehring 2011, 77), whereas in the SNT tropes are mutually rigidly existentially dependent entities. So the nature of tropes is different in this respect in these different accounts. For Campbell and Ehring, they are "junior-substances" (strongly independent particulars); for us they are not. Our theory is a dependence theory, whereas Campbell's and Ehring's accounts are independence theories. So we defend a distinct trope theory for Lowe's criticism. In this respect, the SNT is of the same type as Peter Simons' Nuclear Theory 
(Simons 1994), from which the SNT is, indeed, developed. Our defence is also different from the way Campbell and Ehring could reply to Lowe, since part of it is the analysis of inherence and our analysis must differ from their analyses as for them tropes are junior substances while we maintain them to be rigidly dependent.

${ }^{4}$ The problem of the persistence of tropes overlaps both questions. We have discussed it in Keinänen \& Hakkarainen (2010).

${ }^{5}$ As such, being an individual differs from being a particular, which are distinct category-features of entities. They should not be conflated, for, in principle, universals may be individuals, too: countable entities with identity-conditions (cf., e.g., Lowe's Neo-Aristotelian ontology where kind universals are individual objects). So there are actually three category distinctions involved here: (1) countable vs. not countable, (2) having identity-conditions vs. not having identity-conditions, (3) particular vs. universal. For example, masses such as water may be particulars that are not countable but have identity-conditions (Lowe 2006, 75-8). The exact formulation of the third of these distinctions is the most controversial issue. We do not discuss it in this context as the particularity of tropes is not a topic of this paper. We simply assume that particulars can be exactly similar and still numerically distinct (Maurin 2002, 17, 20-2; cf. Ehring 2011, 30-45). It is a formal category-feature of tropes that they are particulars as well as the feature of universals that they are universals.

${ }^{6}$ Lowe does not use these terms to put the distinction between individuation and identification. His terminological choice is to speak about individuation in a metaphysical and an epistemic sense (Lowe 2003, 75). It corresponds to our distinction between individuation and identification.

${ }^{7}$ Let " $\leq$ " be a relation of improper parthood (cf. Simons $(1987,112)$ for the definition) and "E!" the predicate of (singular) existence. As does Simons (1987, 303), we can define strong rigid dependence as follows: entity $e$ is strongly rigidly dependent on $f$ iff $\neg(\square \mathrm{E}$ !f $) \wedge \square((\mathrm{E}$ !e $\rightarrow \mathrm{E}$ !f $) \wedge \neg($ $\mathrm{f} \leq \mathrm{e})$ ). Weak rigid dependence is defined by replacing $\neg(\mathrm{f} \leq \mathrm{e})$ with $\neg(\mathrm{f}=\mathrm{e})$. Unlike Lowe's $(1998$, 137) definition (D1) of rigid dependence, Simons' definition of weak rigid depencence rules out the rigid dependence of entities on themselves and on necessary existents. If such cases are not taken into consideration, identity dependence entails weak rigid dependence (cf. Lowe 1998, 149-150).

${ }^{8}$ Lowe $(1998,206)$ makes a stronger claim that a substance as a trope bundle would be identity dependent upon (all of its) constituent tropes but the trope theorist need not accept it. 
${ }^{9}$ Tropes are countable in principle, which does not entail that we are actually able to count them in every case.

${ }^{10}$ Since our account of trope identification relies on the claim that tropes are mutually rigidly dependent entities, it is not available to trope theorists (such as Campbell 1990) who assume that tropes are not strongly rigidly dependent on any entity, cf. section 4 for more discussion.

${ }^{11}$ Cf. Ellis (2001) and Mumford (2006), for defence of these claims. See Keinänen (2011) for a more detailed presentation and defence of the SNT.

${ }^{12}$ In addition to tropes as monadic properties, there are relational tropes. It suffices here that we limit ourselves to tropes as monadic properties.

${ }^{13}$ However, in the SNT, we can introduce relational tropes instantiated by the proper parts of a complex substance to determine its features not determined by features of its proper parts (Keinänen 2011, 447).

${ }^{14}$ As we do not introduce quality tropes, we avoid the specific problems pertaining to their identification: e.g., do spatially extended shape tropes have further shape tropes as their proper parts? Do two distinct macro-objects having the same proper parts possess distinct shape tropes?

15 Lowe, too, admits that an entity can be rigidly dependent on another entity without being identity dependent on it (cf. the example of Socrates and Socrates' life in section 2). According to the SNT, identity dependence can be analyzed in terms of strong rigid dependence. Therefore, the former cannot be used in explaining the latter but the converse holds. SRD but not identity dependence is a primitive formal relation, which contributes to the over-all economy of our position (cf. below).

${ }^{16}$ Although not giving a general analysis of identity dependence, Lowe (2006, sec. 3.1) considers the relation of characterization between a mode and the substance having the mode a primitive formal relation, which explains the identity dependence of the mode upon the substance.

${ }^{17}$ Trope $x$ of kind $\mathrm{P}$ is strongly generically dependent on trope $y$ of kind $\mathrm{R}$, if $\operatorname{SGD}(\mathrm{P}(\mathrm{x}), \mathrm{R}(\mathrm{y})) \equiv$ $\square \forall \mathrm{x} \square(\mathrm{Px} \rightarrow \square(\mathrm{E} ! \mathrm{x} \rightarrow \exists \mathrm{y}(\mathrm{Ry} \wedge \neg(\mathrm{y} \leq \mathrm{x})))) \wedge \diamond \exists \mathrm{x}$ Px $\wedge \neg \square \forall \mathrm{x}$ Rx holds. Cf. Simons (1987, 294 ff.) for further discussion.

${ }^{18}$ We accept the following modified version of Simons' (1987, 322; 1998, 243-244) Conditioning Principle: if the rigid dependencies of tropes belonging to a trope bundle are satisfied by the trope constituents of the bundle, the trope bundle itself forms a strongly independent particular. 
${ }^{19}$ Hence, in the context of the SNT, the obtaining of these formal relations spells out when simple substances are identity dependent on their trope parts. Nevertheless, a full analysis of identity dependence, e.g., of complex substances on their necessary parts, is a more complicated matter.

${ }^{20}$ First, unlike the trope theorists, Lowe denies that property tropes of substances, including the necessary tropes, could be their proper parts (Lowe 2009, sec. 2). Second, Lowe $(1998,151)$ is also sceptical of substances having further substances as their necessary parts.

${ }^{21}$ Here, we rely on that the best a posteriori examples of the highest determinables of the determinate features of simple substances are physical quantities (such as mass, charge and spin). A possible a posteriori objection to the SNT might be that the photon is a simple substance that has only one property. However, it is far from clear that this is indeed the correct ontological account of the photon. Physically, in addition to spin, it has the direction of the spin and energy/relativistic mass. Ontologically, there are more than one option to account for this as the direction of the spin and energy/relativistic mass may be explained metaphysically in more than one way (cf. Morganti 2009, 195 ff.; Simons 1994, 570). Instead, it is much clearer that all the other fundamental particles in the Standard Model bear two properties at least (see Morganti 2009, 197).

${ }^{22}$ Ehring's term is "trope swapping across objects" $(2011,79)$.

${ }^{23}$ In addition to a more detailed account of identity dependence (cf. note 19), the advocate of the SNT must explain away the apparent cases of asymmetric existential dependence in presence of mutual rigid dependence (cf. Fine 1995, 271; Lowe 1998, 145; section 2 above).

${ }^{24}$ Hence, we adopt what Schaffer $(2001,248)$ calls "the standard quantitative individuation principle" (QI): $x$ and y are distinct tropes iff they are primitively quantitatively distinct.

${ }^{25}$ Schaffer has also the more metaphysical objection to the primitive identity of tropes that it is not clear whether its advocate can exclude repeatable entities, i.e., universals. If tropes have a primitive identity, nothing seems to rule it out that, instead of two or several tropes in distinct locations (e.g., two or several redness tropes), there is a single trope having a scattered location (2001, 249). Hence, it would be difficult to distinguish between tropes and universals if it is assumed that particulars, in contrast to universals, cannot have scattered location. It seems to us that here Schaffer conflates individuality and particularity. We also consider Ehring's reply to Schaffer sufficient (Ehring 2011, 91). Still we do not agree with Ehring that a trope can have a scattered location. As we have argued elsewhere, they have a specific location (Keinänen \& Hakkarainen 2010, sec. 2).

${ }^{26}$ Other piling problems, which concern one object, such as identifying its several exactly similar tropes ("perfect piling") or tropes of the same determinable kind ("imperfect piling"), do not rise in the 
SNT (cf. Ehring 2011, 86-91). According to it, as was seen just above, an object has only one trope of each determinable kind. This is a good feature of the SNT.

27 Cf. Simons (1994, 573-4) and Schaffer (2001, 254-6) for discussion of the possible implications of the superposed micro-particles to the trope theory. According to Lowe (1998, $62 \mathrm{ff}$.), the superposed electrons are quasi-individuals, entities having determinate countability but no determinate identity-conditions. 